\title{
La distimia femenina y los fenómenos de amor y desamor.
}

\author{
Female dysthymia and the phenomena of love and heartbreak. \\ José Manuel García Arroyo ${ }^{1, a}$
}

\section{RESUMEN}

Aunque el término distimia fue acuñado por Kahlbaum, su significado actual se inició en 1980, cuando aparece en el Manual Diagnóstico de la Asociación Americana de Psiquiatría (DSM) designando una depresión crónica de leve intensidad, distinguible de la depresión mayor. Objetivo: En este trabajo se estudian los casos de dos mujeres con este diagnóstico (según CIE-10) y se examinan los "componentes subjetivos" que sustentan los síntomas depresivos y que habitualmente no se mencionan en las publicaciones. Material y métodos: Se emplea el "Método de Abordaje de la Subjetividad" (MAS), consistente en realizar entrevistas no-directivas y registrar las expresiones verbales de modo fiel, al tiempo que se prescinde de cualquier alusión a teorías, creencias particulares, juicios de valor, etc. Resultados: Se reconoce en estas pacientes una desinserción sentimental respecto al objeto de amor, un convivir desencantado con sus parejas e imposibilidad de separación, al tiempo que aparecen insidiosamente los síntomas depresivos. Este fenómeno tiene como base la caída del "ideal romántico" al que aspiran, que sostiene sus vidas y que funciona como una "agarradera" o "ancla de personalidad", razones por las que no mejoran su sintomatología. Estas originales apreciaciones cuestionan la noción clásica sobre el duelo. Conclusiones: Para que este tipo de pacientes mejoren sintomáticamente hace falta que hablen y se den cuenta de lo que realmente les está sucediendo. El estudio aquí descrito muestra las coordenadas subjetivas que se requiere conocer para poder conducir una adecuada intervención psicoterapéutica.

PALABRAS CLAVE: Distimia, depresión neurótica, duelo, ideales, objeto de amor, Método de Abordaje de la Subjetividad (MAS).

\section{SUMMARY}

Objectives: Although the term dysthymia was coined by Kahlbaum, its current clinical meaning originated in the 1980s, when it appears in the American Psychiatric Association Diagnostic and Statistical Manual (DSM) designating a chronic mild depression distinguishable from Major Depression Disorder. In this article, the cases of two female patients with this disorder (according to the CIE-10) are presented and the "subjective components" that sustain the depressive symptoms, not usually mentioned in the publications, are examined. Material and methods: The Approach to Subjectivity Method (MAS) was used; it consists of non-directive interviews writing down the patient's exact verbal expressions, and avoiding any allusion to theories, particular beliefs, values, judgments, etc. Results: We concluded that these two women have a sentimental distancing from their love object, even though they are unable to separate from or leave him while depressive symptoms appear in an insidious way. This drives them to fall off the romantic ideal they were looking for as a guide for their lives and an anchor of their personality,

1 Departamento de Psiquiatría, Facultad de Medicina,Universidad de Sevilla. Sevilla, España.

a Médico Psiquiatra; Profesor Contratado Doctor. 
and it can also explain why their symptoms do not improve. This point of view questions to some extent the classic notions about "mourning". Conclusions: To improve symptomatically, the dysthymic patients need to talk about and realize what is really happening to them. What is found here shows the subjective coordinates that are necessary in order to conduct an adequate psychotherapeutic intervention.

KEYWORDS: Dysthymia, neurotic depression, mourning, ideals, love object, Method of Approaching Subjectivity (MAS).

\section{INTRODUCCIÓN}

Inicialmente el término distimia fue empleado por Kahlbaum para referirse a depresiones que no mostraban apariencia melancólica (1); después, comenzó a utilizarse de forma generalizada a partir de la $3^{\mathrm{a}}$ edición del Diagnostic and Stadistical Manual of Mental Disorders, definiendo entonces una entidad nosológica concreta ("trastorno distímico") que sustituía al diagnóstico más común en las consultas psiquiátricas de aquellas fechas: la depresión neurótica (2).

En este contexto, se concibe la distimia como una depresión de curso crónico con un humor alterado casi constante, aunque puede sufrir variaciones anárquicas relacionadas con las circunstancias ambientales (2). No se aprecian en el cuadro síntomas de depresión mayor, aunque pueden acumularse en ella manifestaciones neuróticas (hipocondria, ansiedad, fobias, cavilaciones obsesivas, etc.,) (3-5). Tales características hacen que el cuadro clínico se encuentre mal definido o presente una forma variable, no encajando con la idea que el clínico tiene de una depresión "tipica"; ello conlleva que la expresividad sintomática pueda modificarse con el paso del tiempo $(6,7)$, y que el diagnóstico resulte problemático. Sabemos que el sexo es un factor diferenciador significativo en los trastornos depresivos, afectando más a las mujeres y provocando en ellas más discapacidad $(8,9)$, de ahí que se recozca una mayor frecuencia de la distimia en el sexo femenino $(2,4)$.

En este artículo se estudian los componentes subjetivos que se ponen en juego en la aparición, mantenimiento $\mathrm{y} / \mathrm{o}$ estabilización del síntoma (depresivo) en la distimia. Se trata de los hallazgos obtenidos a partir del análisis llevado a cabo con mujeres que padecen este trastorno, aplicándoles el Método de Abordaje de la Subjetividad (MAS) $(10,11)$, que antes ha sido utilizado con éxito para investigar diferentes patologías (psíquicas y físicas), aportando resultados alentadores (12-18). Como veremos, esta pretensión se encuentra en sintonía con las indagaciones realizadas en este terreno, sobre todo aquellas que aluden a la relación entre las producciones sintomáticas y la personalidad o los conflictos inherentes a la situación vivida $(5,19,20)$. Cualquiera de estos factores podría explicar la inconsistencia clínica del cuadro clínico y la pobre respuesta al tratamiento farmacológico, capaz de desalentar a los psiquiatras (21-23). Basándose en estas características, algunos autores niegan a la distimia una estructura psicopatológica propia, considerándola derivada de otras alteraciones psíquicas (desarrollos anómalos de la personalidad, factores psicosociales, etc.) (1) y, quizás por eso, se han empleado numerosos términos para referirse a ella ("temperamento depresivo" de Kraepelin, "disforia histeroide" de Liebowitz y Klein, "depresión caracterológica” de Akiskal, etc.).

Con la presente aportación esperamos arrojar un poco de luz a un asunto tan espinoso, pues el paso de la "distimia" a la "depresión neurótica" ha logrado enfatizar los aspectos descriptivos, los cuáles han quedado por encima de los etiológicos, ganándose en superficie ("extensión") pero perdiéndose en profundidad ("intensión") y no resolviéndose la cuestión sobre cómo intervenir de manera efectiva. La indiscutible indicación psicoterapeútica en estos casos implica tener muy claros los elementos que pueden aparecer durante el proceso y con qué dificultades va a encontrarse el médico cuando realiza la psicoterapia; son estos aspectos los que van a quedar reflejados aquí para así poder ganar en profundidad y facilitar el manejo de las pacientes $(5,19,20)$.

\section{MATERIAL Y MÉTODOS}

El material está formado por un conjunto de mujeres (total: 32), diagnosticadas de "distimia", según los criterios diagnósticos de la CIE-10 (24), y que acudieron a consultar a la Asociación de Psicopatología y Psicoanálisis de Sevilla (APPS). Cualquiera de 
ellas presentaba un síndrome depresivo de larga evolución, sin síntomas psicóticos, de curso tórpido y con poca mejoría con el tratamiento farmacológico. $\mathrm{Al}$ no ser posible mostrar aquí todos los casos, hemos seleccionado dos de ellos, teniendo en cuenta que los resultados obtenidos han sido constatados en los restantes. Seguidamente presentamos una breve historia de cada paciente:

\section{Paciente $1(P-1)$}

Mujer de 52 años, secretaria de un alto cargo en una empresa estatal y casada con un hijo. Se presenta triste, desanimada, agotada y nerviosa, pero continúa trabajando. Lleva varios años con este cuadro, exhibiendo cierta mejoría solo en momentos puntuales, a pesar del tratamiento que tiene prescrito. Participó en una terapia de grupo, pero no pudo resistirlo. Se considera una persona que "se toma todo muy a pecho" y que "se preocupa mucho por todo". De joven se recuerda muy alegre, divertida, sociable y algo coqueta.

\section{Paciente 2 (P-2)}

Mujer de 43 años, casada desde hace 13 años, con dos hijos. Estudió derecho y actualmente regenta su propio negocio. Se presenta desanimada, con llanto fácil y ansiosa; no encuentra mejoría en ningún momento del día. No ve salida a su problema, aunque tampoco puede decir exactamente cuál es. Sin ganas de salir, pero si alguna amiga la llama y sale, puede encontrarse un poco mejor. Ha seguido tratamiento farmacológico con resultados bastante pobres. El trabajo lo realiza con mucho esfuerzo y se considera muy unida a su familia de origen, habiendo sufrido bastante cuando tuvo que separarse de ésta para estudiar la carrera fuera del pueblo.

La investigación que se realiza con estas mujeres no una mera descripción, como se ha venido haciendo, sino que pretende saber qué les sucede realmente y la única forma conocida de lograrlo es estimularlas a que desplieguen la palabra para que, a través de ella, muestren cuáles son sus problemas. El "Método de Abordaje de la Subjetividad" (MAS) cumple esta función, pues consiste en realizar entrevistas nodirectivas en las que se recogen aquellos enunciados que dan cuenta de los procesos interiores $(10,11)$. El entrevistador debe tener una actitud de escucha atenta, lo que consigue dejando de la lado todo aquello que no sea pertinente a la propia situación (preocupaciones, creencias personales, opiniones, etc.); a esto lo hemos llamado "actitud descontaminante" $(10,11)$. En este ambiente, carente de cualquier censura o crítica, se obtiene un material verbal espontáneo y no influido por el investigador que refleja las circunstancias internas correlativas a las producciones sintomáticas. En la distimia esta labor se ve facilitada extraordinariamente debido al predominio en ella de los síntomas psicológicos (y no biológicos) y a la alta capacidad de expresión verbal de las pacientes.

No todas las frases registradas tienen interés en el estudio, dado que las pacientes producen abundante material verbal de deshecho, debido a razones múltiples (necesidad de agradar, justificaciones, quejas, etc). De ahí que hayamos dividido los enunciados obtenidos en "útiles" (o "tipo I") e "inútiles" (o "tipo II") $(10,11)$. Son los primeros los que aportan los datos claves del problema mostrándose, por lo general, de forma aleatoria, aunque algunas veces pueden surgir tras alguna intervención táctica (p. ej. especificándole que no se la va a juzgar). En el Esquema I se muestran las diferencias entre enunciados "tipo I" y "tipo II".

El problema sometido a estudio es complejo en sí mismo pero, al hablar del mismo, las pacientes, sin proponérselo lo descomponen en distintos ennciados ("tipo I"), donde cada uno de ellos revela un aspecto particular del asunto. El material registrado tiene las características de: a) ser privado y no presentarse en otros contextos fuera de la consulta y b) pasar desapercibido en las entrevistas clínicas convencionales de carácter directivo, en las se observa solo lo más evidente, que es hacia donde van dirigidas las preguntas.

Una vez obtenidos los enunciados "tipo I" sorprende la gran similitud que muestran en distintas pacientes; que así sea facilita el agrupamiento de aquellos que son semejantes ("enunciados convergentes") $(10,11)$. Finalmente, con los que presentan el mismo contenido se forman categorías y son estas las que muestran los componentes subjetivos del problema sometido a análisis $(10,11)$. Seguidamente mostramos las categorías halladas tras agrupar los enunciados tomados de las dos pacientes ( $\mathrm{P}-1$ y $\mathrm{P}-2)$, considerando que en los casos no expuestos se han producido verbalizaciones parecidas.

\section{RESULTADOS}

El inicio de las entrevistas evidencia que estas mujeres se encuentran en el desconocimiento más absoluto de qué les ocurre, achacando su estado a 
cuestiones baladíes (p. ej. las condiciones atmosféricas) o a aspectos biológicos de diferente envergadura ( $\mathrm{p}$. ej. genética, hormonas, menopausia, ...). Más tarde, ellas mismas se aperciben de que tales expresiones les sirven para no plantearse las circunstancias reales que motivan su dolor, pues éstas les resultan sumamente penosas de (re)conocer.

La obtención de un material que aporta auténticas pistas sobre el origen del padecimiento no es fácil de lograr pero, con ciertas intervenciones técnicas, se producen cambios favorables que llevan a la consecución de nuevos elementos que tienen como referente las dificultades y/o circunstancias personales. Procediendo así, se muestran una serie de componentes subjetivos de gran valor, teniendo en cuenta que la presentación que hacemos coincide con el orden de aparición durante las entrevistas.

\section{Descubrimiento de un conflicto (crónico) de relación}

El desarrollo de las entrevistas permite a la paciente tomar contacto con algo que, en principio, no podía aceptar: la existencia de un conflicto de pareja de carácter grave y eso la llena de desasosiego; este hallazgo no se realiza de una sola vez, sino de modo progresivo. Al mismo tiempo, expresa una gran rabia y resentimiento hacia su compañero sentimental y, a tanto llega, que situa el problema en él y no en si misma. Digamos que las distimicas suelen colocarse en el papel de víctimas y, en consonancia, registramos muchas oraciones que tienen como sujeto a sus compañeros:

P-1."Creo que me voy enterando un poco más de por qué he estado tan hecha polvo hasta ahora: no soporto a mi marido. Es un cabrón y no tiene remedio". "La vida sin este tío sería mucho más sencilla para mi".

P-2. "Ahora sé un poco más lo que me ocurre y le diré que mi marido es el culpable de todo lo que a mí me pasa. Ahora ya no lo aguanto". "He actuado desde que me casé haciendo todo lo que él quería, pero eso se ha acabado ya. ¿Acaso se merece todo lo que he hecho por él? Yo creo que no".

Cuando se hallan en este momento, lo que impera es la descarga emocional, convirtiéndose el consultorio en un lugar de abreacción aliviadora de tensiones. En consecuencia, salen más tranquilas, al tiempo que se hallan preocupadas por tomar conciencia de un problema hasta entonces no verbalizado y, por lo tanto, desconocido.

\section{Reconocimiento de un desengaño}

Tras un periodo de tiempo variable según el caso ("tiempo subjetivo"), la consultante es capaz de aceptar que el problema se encuentra en ella, más que en su compañero sentimental. Es un momento estelar en el que se acepta la existencia de una desilusión respecto al "objeto de amor".

P-1. "Esto es un gran disgusto para mí (llora) ... porque ha habido un punto de mi vida conyugal en el que no quería estar intimamente con él, usted ya me entiende. Hubo un momento en el que ya no lo quería, me parece a mí, pero no sabría definir exactamente cuando fue. ¿Me he desencantado de mi pareja? No sé, pero yo diría que sí".

P-2."Pienso que todos mis pilares se desajustaron cuando me di cuenta de que él no era exactamente como yo creía, pero esto no lo he podido saber hasta ahora. He sido ignorante de muchas cosas... No sé si saber esto me va a ayudar".

Tarde o temprano, el compañero no va a cubrir las expectativas de la distímica, pasando al polo de la denigración y al desencadenamiento de la agresión que, como hemos observado, se dirige hacia él, a quien hace responsable de la desilusión por no comportarse como esperaba. No es raro encontrar ciertos matices paranoides en el contacto (P-1. "Siempre con lo mismo, él solo quiere hacer eso. Yo no lo aguanto"; P-2. "No me deja en paz, me da la lata y está siempre pendiente de mí").

El efecto clínico-sintomático de este fenómeno (de desencanto) es el humor disfórico, reconocido por casi todos los autores como propio de estas depresiones y casi inexistente en las formas recurrentes $(4,25)$.

\section{La separación (sentimental) del objeto}

Se identifica, a lo largo del proceso, que se ha producido una desinserción sentimental de la pareja. No se trata de un momento puntual, sino de un periodo más o menos prolongado de tiempo que explica, en la mayor parte de los casos estudiados, el inicio insidioso de los síntomas depresivos.

P-1. "Estuve bastante mal, pero ahora sé por qué. Le he dado a él muchas patadas, pero ahora reconozco que estamos juntos casi por estar; pienso que la pasión que existía entre nosotros se acabó hace tiempo. A mí 
él me ha dado asco todos estos años, cada vez que se rozaba conmigo en la cama lo que sentía era asco".

P-2."He ido dándome cuenta de las carencias afectivas que ha tenido mi relación en estos años; hemos perdido la comunicación y la confianza. Creo que actualmente no somos una pareja, hemos fingido que lo éramos. Esto es bastante triste, porque yo me casé con muchísima ilusión y ¡total para nada!”.

Resulta curioso contemplar cómo estas mujeres, a pesar del "divorcio sentimental" al que se encuentran abocadas, no suelen separarse de sus compañeros. Se trata de un hecho singular que pone en cuestión la idea generalmente admitida acerca del duelo. Este último fue estudiado por Freud desde los comienzos de sus teorizaciones, pero matizado y definido en 1915, a propósito de Duelo y melancolía; se entiende en este texto como: "un proceso consistente en desprenderse de un objeto sobre el cuál los actos de amor ya no pueden efectuarse más" (26).

Para comprender la situación de la mujer que pasa por este trance es preciso diferenciar entre "separación física" y "sentimental", ya que ambas no tienen por qué ir unidas. Cuando se habla de "duelo", incluso en las modernas clasificaciones $(24,27)$, la referencia es la primera de ellas, pero no suele tenerse en cuenta la "separación sentimental" sin "separación física", que es al punto al que hemos llegado. En esta coyuntura, la desunión afectiva no es reconocida ni por la paciente ni por el profesional que la atiende, ya que se trata de un fenómeno que solo puede ponerse de manifiesto en las entrevistas no-directivas. Dicho de otra manera, pasa completamente desapercibida en las entrevistas clínicas convencionales debido, entre otras razones, a que lo evidente de la definición de "duelo" no se produce: la "separación física" (muerte, divorcio, etc.).

Las molestias psíquicas de la distímica hay que conectarlas con el "cadáver de relación" que están viviendo: dos personas separadas sentimentalmente que continúan juntas, pudiéndose calificar de "soledad de dos en compañía". Muchos son los signos que se muestran ad oculos en este sentido: falta de comunicación efectiva, inexistencia de acuerdos y funcionamiento independiente, evitación de la proximidad, distanciamiento de las relaciones sexuales, etc. Precisamente, la dedicación al trabajo de la que hablan algunos textos se conecta con la evitación de las conversaciones y/o proximidad afectiva (3). Se añade que, la no-separación puede ser racionalizada de modo conveniente y de muchos modos (no podré mantenerme económicamente, con esta edad ya no podré rehacer mi vida, me verán como una fracasada, le daría un disgusto a mi familia, etc.).

\section{La idealización de la relación sentimental}

Las personas estudiadas idealizan la relación sentimental hasta el extremo, presentando inicialmente grandes expectativas sobre la misma, hasta el punto de que ésta se constituye en la "tabla de salvación" de sus vidas (salir de casa, ser independientes, cambiar psicológicamente, dejar atrás un pasado lleno de frustraciones, etc.). No es extraño que, cuando encuentran a su potencial pareja, se llenan de una ilusión arrebatadora que se transforma, más tarde, en una desilusión proporcional al grado de idealización previo.

P-1."Siempre aposté fuerte por los valores de la pareja. Yo me casé bastante enamorada, pero ahora veo que con él me fabriqué mi propia realidad". "Siempre he estado batallando entre mi deseo y la realidad y debo reconocer ahora que yo a él no lo conocía".

P-2. "Cuando conocí a L. me sentí bastante dichosa, pues pensaba que era el hombre de mi vida, mi media naranja, como se suele decir. Creía que todos mis problemas se iban a resolver casándome, ya no me sentiría jamás desgraciada. Yo soy una mujer bastante apasionada, lo mio son las pasiones, y creo que busqué un amor de cuento".

Hemos constatado, además, que se vuelven tremendamente exigentes con sus compañeros, no permitiéndoles desviarse lo más mínimo; evidentemente, para ellos es imposible complacerlas, aunque lo deseen. Dado que ningún marido puede ser perfecto, con el correr del tiempo, van mostrando los correspondientes fallos y entonces pierden valencias ante ellas, hasta caer vertiginosamente. Ese es el momento en que la paciente pasa a la "denigración"que explica el humor disfórico que presentan (P-1. "Me encuentro siempre enfadada, mi hijo me dice que estoy inaguantable". "Mi hijo se va el fin de semana fuera de casa para no aguantarme y yo lo comprendo").

Antes de que se produzca la caída del consorte no es extraño hallar intentos desesperados de la paciente para modificar a su antojo la personalidad de aquel, con la idea de que encaje adecuadamente en la estructura mental que tiene, sin importarle nada lo que él sienta. Hallamos aquí la fuerte ambivalencia de la distímica, que se encuentra relacionada con la mayor 
o menor distancia de su pareja respecto al "ideal" al que aspira. De esto se sigue que, en realidad no conoce a su compañero cuando decide convivir con él y aquello que no sabe lo rellena con fantasías $(28,29)$. El desconocimiento crónico de la persona a quién se unen, junto con el desprecio que sienten hacia él, les impide establecer nuevos pactos y alianzas. Akiskal (4) acertadamente, ha señalado que: "si están casados, están atrapados en matrimonios infelices que no conducen ni a la reconciliación ni a la separación".

\section{¿Qué pierde realmente la distímica?}

Pareciera, con lo que hemos expuesto, que la distímica muestra un problema de desamor que puede resolverse simplemente con la separación y el cambio de pareja; no se trata solo de eso, porque entonces el cuadro depresivo sería pasajero. La pérdida de estas mujeres va mucho más allá, es más profunda, pues se encuentra en el "ideal romántico" y esto puede verse claramente en cómo se hallan desmotivadas ante cualquier posible relación, tanto con la pareja actual como con cualquiera que se les presente:

P-1."No sé, pero pienso ahora que no voy a poder querer a ninguna persona. Si yo me separara de mi marido, pienso que no podría enamorarme ya de nadie".

P-2. "De siempre he sido una persona enamoradiza, pero ahora creo que no podría llegar a amar a ningún hombre, no solo a mi marido, sino a cualquiera aunque fuera el hombre más lindo del mundo”.

Esto es exactamente lo que las lleva a no separarse, ya que no tienen la esperanza de volver a unirse afectivamente a alguien con la fuerza que esperan. Por consiguiente, la pérdida de las distímicas no es "externa" sino "interna" y va más allá de la simple relación, pues se dirige a una de las "agarraderas" o de los "pilares" básicos de la personalidad, que ellas definen de distintas maneras: el "amor de cuento", el "príncipe que me iba a salvar", "aquel hombre que me trataría como a una reina", "el amor de mi vida" o "el hombre eternamente enamorado".

Este "ideal romántico" ha caído debido a los continuos choques con la realidad; en efecto, sus parejas se comportaban como humanos, presentando defectos e incluso, algunos de ellos que pudimos entrevistar eran personas bastante limitadas. Pero, llega a más: en muchas mujeres estudiadas descubrimos que querían ser adoradas por sus maridos de una forma incondicional y de un modo continuo, sin ellas dar nada a cambio.
P-1."El no me ha respondido como yo esperaba, no me ha dado el cariño que yo necesitaba". "Mi marido es una persona que en la casa le gusta hacer bricolaje y se ha llevado mucho tiempo encerrado en su cuarto haciendo tonterías, mientras tanto yo me he sentido muy sola y esto ¡durante tantos años! Ha acabado todo por romperse, no me extraña". "Por todo lo que he dicho, yo creo que mi marido ha sido siempre un apático".

P-2."Este tío va a su bola y a mí ni me echa cuenta ni me atiende. Debería haber estado más pendiente de mí. Yo no quiero una persona así a mi lado". "Yo quería junto a mí a una persona muy cariñosa y tierna conmigo y eso no lo he tenido".

Llegamos a la conclusión de que estas pacientes más que "amar al hombre" se puede decir que "aman al amor" y, por si fuera poco, aquellas cosas que poseen (trabajo, hijos, amistades, etc.) y que podrían haberlas ayudado en la situación depresiva que atraviesan, no les sirven de mucho. Algunas pueden serles útiles para desconectarse (temporalmente) de la imponente presencia de los síntomas; de ahí lo influenciables que son por las condiciones externas (3), dándose la posibilidad de obtener cierto placer en algunas actividades tras un esfuerzo inicial (30), aspecto al que Klein se refiere con el término de "anhedonia parcial" (31). Afirmamos, en definitiva, que nada puede ya taponar el vacío al que se ven abocadas tras la deconstrucción del "ideal romántico" que las sostenía.

\section{DISCUSIÓN}

La "distimia" es la categoría heredera de la "depresión neurótica", un cambio de denominación que no ha supuesto transformación alguna en los aspectos epidemiológicos, ya que sigue siendo uno de los diagnósticos más frecuentes de la psiquiatría actual. Este hecho junto a la mayor frecuencia en mujeres y a la resistencia que presenta a los tratamientos psicofarmacológicos, ha sido el motor de esta investigación. Además, la distimia ha sido muy poco estudiada pues la mayor parte de las investigaciones se han centrado en la depresión mayor (32), a pesar de considerarse que la distimia puede tener un peor pronóstico que esta última (33) y que es capaz de producir igual o mayor limitación funcional $(34,35)$.

El estudio de un grupo de mujeres distímicas, buscando qué les sucede más allá de lo que aportan los criterios diagnósticos $(24,27)$, que se quedan en la superficie, brinda la oportunidad de conocer los "componentes subjetivos" del trastorno. Se trata de 
una verdadera adquisición, pues con ellos pueden sentarse las bases de un tratamiento psicoterapéutico reglado, lo cuál posibilita avanzar en el trabajo que se está realizando actualmente con las pacientes. Añadimos que, tradicionalmente, se les negó la palabra a las mujeres porque supuestamente eran "demasiado emocionales" y las emociones perturbaban la "racionalidad" y la "claridad de conciencia" que permitían el pensar, hasta que Freud sentó las bases de la escucha a este colectivo; basta recordar que comenzó su andadura tratando el malestar femenino afiliado al terreno de la conversión (36). Justamente, esta es la linea aquí seguida, reafirmándonos con Freud en mostrar un interés de atender y valorar aquello que las mujeres tienen que decir.

Después de realizar con ellas una escucha libre, para no caer en lo que modernamente se denominan "prejuicios cognitivos" o "falsificaciones de los resultados provocadas por quién realiza la observación", descubrimos una importante pérdida que no es un simple desapego sentimental sino que va mucho más allá, lo que explica la persistencia del cuadro depresivo. Aquello que pierden es el "ideal romántico", que las mantiene vivas e interesadas en existir; se desinstala entonces un "principio rector" que, una vez cede, lleva a su portadora a mostrarse sin ilusiones por cuanto le rodea. Se suma a ello, que son personas que se juegan la vida "a una sola carta", la del "amor", pues no parecen interesarse seriamente por ninguna otra cosa $\mathrm{y}$, si acaban perdiéndolo, se desploma la estructura psíquica. Este fenómeno puede ser demoledor, incluso mucho antes de que aparezcan los síntomas (depresivos). A título de ejemplo, atendemos actualmente a una chica joven que lleva el "camino" de la distimia y que pronuncia en la consulta una demoledora frase que puede sellar su destino: "A mí realmente no me interesa nada, ni siquiera mi carrera, yo solo quiero tener un novio y entonces seré feliz".

Los fenómenos registrados no pueden verse en las entrevistas convencionales debido a un doble prejuicio en la escucha, consistente en: $1^{\circ}$ ) las cuestiones relativas a los sentimientos amorosos son entendidas como "pamplinas", que no pueden deprimir a nadie teniendo, en cambio, un gran peso las modificaciones biológicas, aunque sean supuestas (en la distimia) y $2^{\circ}$ ) se cree que toda pérdida tiene que ser necesariamente "externa" (separación, divorcio, muerte,...) y no "interna", como es el caso que ahora nos ocupa y que cambia nuestras coordenadas sobre la concepción que tenemos del duelo. Este último se ha entendido, en el sentido clásico, como una pérdida "externa", referida a una persona (amada) y el correspondiente esfuerzo en tiempo y energía para adaptarse a la desaparición del objeto ("trabajo de duelo") (26).

El duelo que aquí se expone es más complejo porque la pérdida no es "externa", ya que la persona sigue unida al objeto de amor, no existiendo una ruptura con él, pero eso no significa que no exista. La pérdida ha sido constatada en las distímicas analizadas, pero tiene las siguientes particularidades: a) no es vivenciada por ellas, b) es interna, pues se rompe el vínculo afectivo con la pareja y c) solo puede evidenciarse en entrevistas en las que se les deje hablar libremente, sin buscar el cumplimiento de ningún criterio diagnóstico, como es el caso de una investigación reglada o en el contexto de un proceso psicoterapéutico. Estas características nos llevaron a establecer "dos tipos de duelo" fácilmente identificables en la clínica para quién quiera (o pueda) verlos (37).

Estamos ante un asunto que aporta pistas al médico en sus actuaciones ya que, observando el conflicto de pareja que tienen, muchas veces se les da consejos para que se separen, tratándose de una recomendación claramente errada. Esto se debe a que el distanciamiento físico no arregla absolutamente nada, pues terminan desilusionadas no solo de sus parejas, sino del mismo "amor", debido a esperan demasiado y ningún hombre puede cumplir la adoración incondicional a la que aspiran incansablemente. Entonces, se llenan de desazón al comprobar la imposibilidad de alcanzar el "amor de cuento" o de "película romántica" en el que se sostienen y, en consecuencia, la vida acaba perdiendo su sentido.

Se demuestra que la no-separación a la que se ven abocadas se sostiene en poderosas razones subjetivas, que han sido identificadas en la investigación: a) no creen en la posibilidad de reanudar su vida amorosa con una persona nueva, b) la separación implica concienciarse del hundimiento del "gran proyecto" de sus vidas, c) pueden ser objeto de crítica ajena o de proyectar fuera la imagen de "fracasadas", lo que puede relacionarse con lo influenciables que son y d) dar el paso significa provocar un gran conflicto familiar por el que no están dispuestas a pasar.

En el fondo hemos de reconocer en estas mujeres una "incapacidad de amar" neurótica, a la que hace tiempo dimos el nombre de "anagapia" (38); esto es obvio, pues cabe preguntarse: ¿cómo es posible amar a alguien a quién no se conoce realmente? Se añade 
que, a medida que la relación evoluciona, las cosas van a peor, funcionando en el registro de: "si no tengo el amor maravilloso que deseaba, no tendré ningún amor". Ello demuestra, a las claras, que "interesarse por el amor" no es lo mismo que tener "capacidad de amar" o "interesarse por una persona concreta".

Aunque pueda parecer incongruente, la paciente "prefiere" instalarse en el dolor psíquico antes que reconocer la "desilusión-separación-desconfiguración" que la embarga, ya que se trata de algo sumamente penoso que termina con las ilusiones creadas durante años. De ahí que muchas distímicas indiquen, a lo largo del proceso, que la depresión es para ellas como un "refugio", prefiriendo el estancamiento vital en el que se encuentran antes que progresar reconociendo su propio fracaso. Este asunto, que parece imverosímil pero que es demostrable clínicamente, no escapó a la gran intuición de Victor Hugo, cuando habló de la "felicidad de estar triste".

Los resultados obtenidos no terminan la investigación sobre la distimia femenina, siendo necesario trabajar con nuevos casos para comprobar si los aspectos subjetivos hallados de estas pacientes se mantienen. Al mismo tiempo, pueden abrirse nuevas vías y una de ellas podría consistir en plantearse si es posible que la dinámica desvelada en este trabajo se relaciona con algún trastorno concreto de la personalidad, lo que llevaría (en un próximo estudio) a procurar aclarar algo sobre las relaciones entre la personalidad y la distimia, asunto que está dando muchos quebraderos de cabeza a los psiquiatras $(39,40)$, donde algunos indican que la patología aquí analizada sería secundaria a un trastorno de la personalidad (41).

Conflictos de interés: EL autor declara no tenerlos.

Declaración de financiamiento: Financiado por el autor.

\section{Correspondencia:}

José Manuel García Arroyo.

c/ Luis Montoto $n^{\circ} 83-3^{\circ} \mathrm{C}$.

41.018. Sevilla, España.

Correo electrónico: jmgarroyo@us.es

\section{REFERENCIAS BIBLIOGRÁFICAS}

1. Gastó C, Vallejo J. Aspectos históricos de la depresión. En: Vallejo J, Gastó C. Trastornos afectivos: ansiedad y depresión. Barcelona: Masson; 1999.
2. Kaplan HL, Sadock BJ. Sinopsis de Psiquiatría. Madrid: Wolters Kluwer; 2015.

3. Vallejo J, Menchón JM. Distimia y otras depresiones no melancólicas. En: Vallejo J, Gastó C. Trastornos afectivos: ansiedad y depresión. Barcelona: Masson, 1999.

4. Akiskal HS. Distimia, ciclotimia y otros trastornos del humor subclínicos crónicos. En: Gelder MG, López Ibor JJ, Andreasen N. Tratado de Psiquiatría. Barcelona: Ars Médica; 2002.

5. Menchón JM, Vallejo J. Distimia. En: Roca M. (coord.). Trastornos del humor. Madrid: Editorial Panamericana; 1999.

6. Akiskal HS, Bitar A, Puzzantian V. The nosological status of neurotic depression. Arch Gen. Psychiatry. 1978; 35: 756-766.

7. Akiskal HS. Dysthymic disorder psychopathology of proposed chronic depressive subtypes. Am J Psychiatry. 1983; 140: 11-20.

8. Roca M, Baca E, Prieto R, García-Calvo C. Diferencias de género en el perfil clínico, respuesta y remisión de pacientes depresivos tratados con venlafaxina retard. Actas Esp Psiquiatr. 2008; 36 (2): 82-89.

9. García MP. Depresión y perimenopausia: una revisión. Actas Esp Psiquiat. 2009; 37(4): 213-221.

10. García JM, Domínguez ML. ¿Por dónde seguir investigando en psicopatología? Informaciones Psiquiátricas. 2010; 1(199): 39-56.

11. García JM. El cáncer de mama: metodología de la subjetividad. Sevilla: Punto Rojo; 2016.

12. García JM, Domínguez ML. El dolor: una encrucijada entre el cuerpo y la subjetividad ( $1^{\text {a }}$ parte). ALCMEÓN. 2009; 16(1): 26-37.

13. García JM, Domínguez ML. El dolor: una encrucijada entre el cuerpo y la subjetividad ( $2^{\mathrm{a}}$ parte). ALCMEÓN. 2009; 16(1): 38-48.

14. García JM, Domínguez ML, Fernández-Argüelles P. ¿Qué desvela la psicoterapia sobre el paciente hipocondríaco? Psicologia.com. 2011; 15:55. Handle: http://hdl.handle.net/10401/4665

15. García JM, Domínguez ML. Subjective aspects of Burnout Syndrome in the Medical Profession. Psychology. 2014; 5(18): 2064-2072.

16. García JM, Domínguez ML. Aspectos psicológicos de la mujer diagnosticada de cáncer de mama. Ginecología y Obstetricia Clínica. 2010; 11(4): 190198.

17. García JM, Domínguez ML. Psychological problems derived from mastectomy: A qualitative study. International Journal of Surgical Oncology. 2011; 2011: 132461. doi: http://dx.doi. org/10.1155/2011/132461

18. García JM. Aspectos subjetivos de la mujer con dismenorrea primaria. Revista Chilena de Obstetricia y Ginecología. 2017; 82(3): 271-279.

19. Klerman GL, Endicott J, Spitzer RL, Hirschfeld 
RMA. Neurotic depressions: a systematic analysis of multiple criteria and meaning. Am J Psychiatry.1979; 136: 57-61.

20. Kocsis JH, Frances A. A critical discussion of DSMIII dysthymic disorder. Am J Psychiatry. 1987; 144: 1534-1542.

21. Tyrer P. Neurosis divisible? Lancet. 1985; 1: 685688.

22. Tyrer P, Alexander J, Remington M, Riley P. Relationship between neurotic symptoms and neurotic diagnosis. J Affect Disord. 1987; 13: 13-21.

23. Tyrer P, Seivewright N, Ferguson B, Tyrer J. The general neurotic syndrome: a coaxial diagnosis of anxiety-depression ad personality disorder. Acta Psychiat Scand. 1992; 85: 201-206.

24. World Health Organization. International classification of diseases an related health problemas. Geneva: World Health Organization; 2007.

25. Hamilton M. Frequency of symptoms in melancholia (depressive illness). Br J Psychiatry.1989; 154: 201206.

26. Freud S. Duelo y melancolía. En:Freud S. Obras Completas (vol. 2). Madrid: Biblioteca Nueva; 1981.

27. American Psychiatric Association. Diagnostic and Statistical Manual of Mental Disorders. Arlington, VA: American Psychiatric Publishing; 2013.

28. García JM. Psicología y Psicopatología de la fantasía. ALCMEÓN. 2013; 18(3): 175-186.

29. García JM. Psicología y Psicopatología de la fantasía. ALCMEÓN. 2013; 18(3): 187-200.

30. Loas G, Boyer P. Anhedonia in endogenomorphic depression. Psych Res. 1996; 60: 57-65.

31. Klein D. Endogenomorphic depression. Arch Gen Psychiatry. 1974; 31: 447-454.
32. Airaksinen E, Larsson M, Lundberg I, Forsell Y. Cognitive functions in depressive disorders: evidence from a population-based study. Psychol Med. 2004; 34: 83-91.

33. Gureje O. Dysthymia in a cross-cultural perspective. Cur Opin Psych. 2010; 24: 67-71.

34. Aragonés E, Gutiérrez M, Pino M, Lucena M, Cervera J, Garreta I. Prevalencia y características de la depresión mayor y de la distimia en atención primaria. Aten Primaria. 2001; 27: 623-628.

35. Klein DN, Schatzberg AF, McCullough JP, Keller MB, Dowling F, Goodman D. Early-versus lateonset dysthymic disorder: comparison in out-patients with superimposed major depressive episodes. J Affect Disord. 1999; 52: 187-196.

36. Freud S. Estudios sobre histeria. En: Freud S. Obras Completas (vol. 1). Madrid: Biblioteca Nueva; 1981.

37. García JM. Subjetividad y duelo. Ciencia y Humanismo en la Salud. 2017; 4(3): 87-96.

38. García JM. Análisis fenomenológico de las formas de amor morboso. Granada: Actas del V Congreso Español de la FESS; 1994.

39. Klein D, Schwatz J, Rose S, Leader J. Five-year course and outcome of dysthymic disorder: a prospective, naturalistic follow-up study. Am J Psychiatry. 2000; 157: 931-939.

40. Akiskal H. Dysthymia and cyclothymia in psychiatric practice a century after Kraepelin. J Affect Disorder. 2001; 62: 17-31.

41. Jiménez E, Gallardo G, Villaseñor T, Gonzalez A. La distimia en el contexto clínico. Rev Colomb Psiquiat. 2013; 42(2): 212-218.

Recibido: 26/06/2019

Aceptado: 16/09/2019 